\title{
Characterization and applications of silver nanoparticles-decorated electrospun nanofibers loaded with polyphenolic extract from rambutan (Nepelium lappaceum)
}

\author{
Hilary Urena-Saborio ${ }^{\mathrm{a}}$, Gerardo Rodríguez ${ }^{\mathrm{b}}$, Sergio Madrigal-Carballo ${ }^{\mathrm{c}}$, \\ Sundaram Gunasekaran ${ }^{\mathrm{a}, *}$
}

${ }^{a}$ Department of Biological Systems Engineering, University of Wisconsin-Madison, Madison, WI 53706, USA

${ }^{\mathrm{b}}$ Laboratory of Phytochemistry, National University of Costa Rica, Heredia, 11801, Costa Rica

${ }^{\mathrm{c}}$ Department of Animal Sciences, University of Wisconsin-Madison, Madison, WI 53706, USA

\section{A R T I C L E I N F O}

\section{Keywords:}

Antibacterial

Antioxidant

Bioactive

Electrospun membrane

Nanoparticles

Scaffolds

\begin{abstract}
A B S T R A C T
Rambutan (Nephelium lappaceum) is a traditional fruit originally from Asia and now widely available in Central America. The rind of rambutan is rich in antioxidants, especially polyphenols, which have natural antiinflammatory and antimicrobial properties. Rambutan polyphenolic extract (RPE) was encapsulated in multicomponent electrospun nanofiber (ESNF) templates. These nanofibers were decorated with silver nanoparticles (AgNPs) via in situ electrochemical reduction. The RPE-loaded and AgNPs-decorated ESNF (AgNPs/RPE-ESNF) were evaluated for their hydrophilic, antioxidant, and antimicrobial properties. Results indicate that the addition of RPE to synthetic polycaprolactone (PCL) ESNF improves hydrophilicity and cell attachment and proliferation in vitro. In addition, a synergistic antibacterial and cell proliferation effect between AgNPs and RPE was observed when they were combined in the multi-functionalized ESNF system. The effectiveness of AgNPs/RPE-PCL ESNF as an antioxidant and antibacterial matrix could potentially promote wound healing and also serve as a hybrid scaffold for tissue engineering.
\end{abstract}

\section{Introduction}

Bioactive compounds in plant extracts such as vitamin C, vitamin $\mathrm{E}$, xanthophylls, carotenes, tannins, and phenolics are widely used in ethnomedicines in many countries around the world, as they are well known for their antioxidant, antibacterial, and anti-inflammatory properties [1-3]. In addition, plant extracts can reinforce biopolymers by modifying their biological activity [4,5], physicochemical properties and further extend their applications $[6,7]$.

Various techniques have been investigated to retain and/or enhance the functionalities of plant extracts when used in conjunction with biopolymer composites. Among the many systems, electrospinning approaches are simple, versatile, and cost-effective [8-10]. Electrospinning also allows developing multi-component biomaterials loaded with active ingredients and tailored functionalities on electrospun nanofibers (ESNF) [11].

Rambutan (Nephelium lappaceum L. family Sapindaceae) is an attractive tropical fruit originally from Southeast Asia [12]. The peel or rind of rambutan contains antioxidants and antimicrobial agents. Some rambutan varieties that meet quality characteristics include Rongrein, R-134,
Criollo, R-162 and R-167, since they have been improved in terms of color, flavor, texture, fruit size, and pulp detachment ability [13]. Chemical compounds found in rambutan peel include anthocyanins, phenolics and flavonoids. Geraniin, an ellagitannin, has been identified as the primary compound in the ethanolic extracts of rambutan rind. This compound possesses a wide range of bioactive properties including antioxidant, free radical scavenging, anticancer, antimicrobial, antiviral, and anti-hyperglycemic activities. Corilagin, ellagic acid, and gallic acid are the primary metabolites of geraniin $[14,15]$. Liang et al. [16] have isolated five oleane-type triterpene oligoglycosides from rambutan peel.

Electrospinning allows the fabrication of nanofiber membranes loaded with bioactive plant-based extracts as functionalizing agents to improve biocompatibility and antioxidant properties of synthetic ESNF. Decoration of rambutan polyphenol extract (RPE)-loaded ESNF with silver nanoparticles (AgNPs), which have proven antibacterial abilities [17], provides a multi-component template with synergistic antibacterial and antioxidant properties. AgNPs can release silver ions that are effective against bacteria resistant to antibiotics, as well as against fungi and viruses [18]. Silver ions inhibit phosphate uptake and exchange in bacteria and cause efflux of accumulated phosphate,

\footnotetext{
* Corresponding author.

E-mail address: guna@wisc.edu (S. Gunasekaran).
} 
mannitol, succinate, glutamine, and proline. Silver ions can also interact with the bases in DNA and affect its ability to replicate [19]. The electrospinning affords operational flexibility for incorporating other species in the fibers. Many studies have been carried out incorporating AgNPs in various polymeric fibers by electrospinning, which show antibacterial activity $[20,21]$.

The aim of this study was to develop cost-effective and facile multifunctionalization of ESNF by blending synthetic polycaprolactone (PCL) polymer with rambutan RPE followed by surface decoration of ESNF with AgNPs, targetting improved biocompatibility, cell attachment, antibacterial, and antioxidant properties.

\section{Materials and methods}

\subsection{Materials}

Rambutan samples from grafted varieties (R-134, Rongrein, and Criollo) were obtained from the Laboratory of Phytochemistry (LAFIT) at the School of Chemistry, National University, Costa Rica. Polycaprolactone (PCL, molecular weight $=80 \mathrm{kDa}$ ), acetone, and alamarBlue solution were purchased from Thermo Fisher Scientific (Waltham, MA, USA) and used without further purification.

\subsection{Polyphenol extraction and characterization}

Rambutan peels from different varieties were separately frozen and lyophilized (Labconco 2.5 L plus, Kansas City, MO). The dried samples were ground to $1.0 \mathrm{~mm}$ particle size, and $1.0 \mathrm{~g}$ sample of rambutan powder was mixed with $25 \mathrm{~mL}$ of extracting solution (ethanol: $\mathrm{HCl}, 95: 5$ ) and stirred for $30 \mathrm{~min}$ to obtain crude RPE solutions. The solutions were filtered (Whatman 42, Sigma-Aldrich, St. Louis, MO), concentrated in a rotary evaporator (Buchi R200, New Castle, DE) under reduced pressure $(690 \mathrm{kPa})$ and temperature not higher than $40{ }^{\circ} \mathrm{C}$ for three hours, lyophilized, and stored in a desiccator as crude RPE powder.

\subsection{Electrospinning of crude RPE}

The crude RPE powder was dissolved in methanol $(96 \mathrm{mg} / \mathrm{mL})$ and combined with PCL $(100 \mathrm{mg} / \mathrm{mL})$ in an organic solvent mixture of chloroform and methanol $(9: 1 \mathrm{v} / \mathrm{v})$. The RPE-PCL blend $(5 \mathrm{~mL})$ was prepared by diluting crude RPE solution with PCL to a final RPE concentration of $12 \mathrm{mg} / \mathrm{mL}$. The RPE-PCL blends were characterized by measuring their viscosity by a stress-sweep test in a programmable rheometer (Brookfield DV-III Ultra, UK) at a shear rate of $100 \mathrm{~s}^{-1}$. Their electrical conductivity was measured at $25^{\circ} \mathrm{C}$ with a conductivity meter (Orion Star A215, Thermo Fisher, Waltham, MA) with an electrode conductivity constant of $0.7265 \mathrm{~cm}^{-1}$.

The RPE-PCL solutions were loaded into a $5 \mathrm{~mL}$ syringe fitted with 18-G needle and connected to an electrospinning setup. A 12-kV electric field was applied using a high voltage power source (Gamma High Voltage Research, Ormond Beach, FL). The distance between the needle tip and the aluminum foil-coated copper collector was fixed at $10 \mathrm{~cm}$, creating a $1.2 \mathrm{kV} / \mathrm{cm}$ electric field strength on the RPE-PCL solution. After $3 \mathrm{~mL}$ of the solution was electrospun, the resulting RPE-PCL ESNF mats were placed in a vacuum chamber for $24 \mathrm{~h}$ to remove any residual of organic solvent, and then stored in a desiccator for further experiments.

\subsection{Surface decoration of RPE-PCL ESNF with AgNPs}

AgNPs were directly formed onto the surface of RPE-PCL ESNF mats via a chemical reduction method [22]. RPE-PCL ESNFs were cut into 8-mm diameter circles and dipped into $\mathrm{AgNO}_{3}(100 \mathrm{mM})$ for $5 \mathrm{~min}$ at room temperature. Following this, the ESNF discs were dipped in $\mathrm{NaBH}_{4}$ $(100 \mathrm{mM})$ for $5 \mathrm{~min}$ at room temperature, to reduce silver ions to elemental silver. The spontaneous formation of AgNPs is indicated by the change in color of the ESNF mat from pink to dark brown. Once the reduction reaction was completed, samples were washed thoroughly with deionized water to remove any remaining chemicals or AgNPs formed but were unattached to ESNF. The samples (AgNPs/RPE-PCL ESNF) were dried in a vacuum chamber at room temperature for $12 \mathrm{~h}$ and stored in a desiccator until further analysis. The average hydrodynamic diameter of AgNPs used for surface coating was measured after dipping reaction by dynamic light scattering measurements (NanoBrook 90Plus particle size analyzer, Brookhaven Instruments, Holtsville, NY).

\subsection{Characterization of RPE and AgNPs/RPE ESNF}

The chemical profiles of RPE-PCL ESNF and AgNPs/RPE-PCL ESNF were characterized by attenuated total reflectance Fourier-transform infrared spectroscopy (Nicolet 4700 ATR FT-IR, Thermo Scientific, Grand Island, NY) in the wavenumber range of 950 to $4000 \mathrm{~cm}^{-1}$, and thermogravimetric analysis (TGA, Q100, TA Instruments, Lindon, UT). For TGA, a heating rate of $20{ }^{\circ} \mathrm{C} / \mathrm{min}$ was applied, and the samples were scanned between 50 to $400{ }^{\circ} \mathrm{C}$ under inert atmosphere (nitrogen 20 $\mathrm{mL} / \mathrm{min}$ ). Scanning electron microscopy (SEM, Leo 1530-FE, Zeiss, Cambridge, UK) was used to study the surface morphology of ESNF. Image $\mathrm{J}$ software (NIH, Bethesda, MD) was used to determine the average fiber diameter by measuring at least 20 nanofibers on the SEM micrographs. Statistical analysis was performed using Assistant VR software (Statistics, Arlington, TX).

The swelling property of the nanofibers was studied by immersing the samples in $1 \mathrm{X}$ phosphate buffered saline (PBS) buffer at $37^{\circ} \mathrm{C}$ for 5 , $15,30,45,60,120,240 \mathrm{~min}$, and was calculated as:

Swelling $(\%)=\left(\frac{\mathrm{W}_{\text {Wet }}-\mathrm{W}_{\text {Dry }}}{\mathrm{W}_{\text {Dry }}}\right) \times 100$

Where, $\mathrm{W}_{\text {Dray }}$ and $\mathrm{W}_{\mathrm{Wet}}$ are dry and wet mass, respectively. Pore area of the ESNF membranes was determined by Image $J$ software combined with Diameter J package (LOCI, Madison WI) with the SEM micrographs [23].

\subsection{Determination of RPE release and antioxidant activity}

To simulate the release of RPE at physiological conditions, the samples were placed in PBS buffer, which has osmolarity and ion concentration similar to those in the human body, and a normal body temperature $\left(37^{\circ} \mathrm{C}\right)$. Release of RPEs from RPE-PCL ESNF was measured by immersing 8-mm diameter ESNF discs into $5 \mathrm{~mL} 1 \mathrm{X}$ PBS solution, followed by incubation at $37^{\circ} \mathrm{C}$. One milliliter solution was drawn at different times $(6,12,24,48$ and $72 \mathrm{~h})$, and $1 \mathrm{~mL}$ PBS was returned into each sample container to keep the total volume constant. The released RPEs were quantified by the Folin-Ciocalteu method for their total phenolic content (TPC) [24].

Antioxidant capacity was determined by the DPPH assay. Briefly, a solution of 2,2-diphenyl-1-picrylhydrazyl (DPPH) was prepared as described in Bondet et al. [25] A 96-well plate was filled with a blank solution composed of $30 \mu \mathrm{L}$ of an 80:20 (v/v) methanol:water solution; $30 \mu \mathrm{L}$ of gallic acid $(0.0215 \mathrm{mg} / \mathrm{mL})$. After samples were dipped into the blank solution for $1 \mathrm{~min}, 30 \mu \mathrm{L}$ of sample extract was added into each well, and then $270 \mu \mathrm{L}$ DPPH solution $(0.042 \mathrm{mg} / \mathrm{mL})$ was added to complete the redox reaction. A characteristic color change developed in the samples, after $30 \mathrm{~min}$ of incubation at $25^{\circ} \mathrm{C}$, was monitored using a microplate reader (Synergy HT Multi-Mode, Biotek, Winooski, VT) at a wavelength of $515 \mathrm{~nm}$.

\subsection{Determination of in vitro antibacterial activity}

Microbial susceptibility assay measuring zone-of-inhibition (ZoI) was employed [26] to qualitatively evaluate the antimicrobial activity of RPE-PCL ESNF and AgNPs/RPE-PCL ESNF . Samples were tested against three human pathogens: Escherichia coli ATCC 25992 (Ec), Staphylococcus aureus ATCC 25923 (Sa), and Pseudomonas aeruginosa PAO1 (Pa). 
Whatman sterile filter ( $6 \mathrm{~mm}$ diameter) and $100 \mathrm{mg} / \mathrm{mL}$ PCL served as negative controls, and kanamycin $(6 \mathrm{mg} / \mathrm{mL}$ solution) as positive control. Firstly, a loopful of each tested bacteria was inoculated into $50 \mathrm{~mL}$ of nutrient broth and incubated at $37{ }^{\circ} \mathrm{C}$ overnight. Subsequently, $1 \mathrm{~mL}$ of this culture was diluted to $10^{7} \mathrm{CFU} / \mathrm{mL}$ concentration using 10 -fold dilution method, and then $100 \mu \mathrm{L}$ of diluted solution was spread on nutrient agar plates. After spreading was completed, sterilized (ultraviolet irradiation in a laminar flow hood for $30 \mathrm{~min}$ each side) 8-mm diameter discs of RPE-PCL ESNF and AgNPs/RPE-PCL ESNF were placed on the inoculated nutrient agar plates. Kanamycin $(5 \mu \mathrm{L})$ was added into a sterilized filter disk and placed on the inoculated nutritional agar. The diameter of $\mathrm{ZoI}$ on each inoculated plate was measured after incubation at $37{ }^{\circ} \mathrm{C}$ for $24 \mathrm{~h}$. The tests were carried out in triplicates.

\subsection{Cell adhesion and proliferation assays}

The cell adhesion and proliferation properties of the ESNF mats were tested in vitro using 3T3 mouse embryonic fibroblast cells $[27,28]$. ESNF sample discs prepared as above were placed into different wells in a sterile cell culture plate with media and $3 \mathrm{~mL}$ of fibroblast cell suspension $\left(1.56 \AA \sim 10^{5}\right)$. Firstly, the adhesion assay was carried by the incubation of the cells with the membranes for 40 min with a mild stirring (2 $\mathrm{rpm}$ ) at room temperature. Each membrane was relocated in different wells of the same plate without cell suspension and left incubating for $15 \mathrm{~min}$ at $37{ }^{\circ} \mathrm{C}$ and $5 \% \mathrm{CO}_{2}$, followed by additional $2 \mathrm{~h}$ of incubation after adding alamarBlue cell viability reagent $(10 \mu \mathrm{M})$. The fluorescence of the disc samples was measured at 540/590 nm using a microplate reader (SpectraMax Plus, Molecular Devices, Sunnyvale, CA) as an indicator of overall cell attachment.

After the cell attachment stage was completed, the same sample discs were coated with DMEM culture medium and placed in an incubator at $37{ }^{\circ} \mathrm{C}$ for 3,7 , and 14 days for cell proliferation. After each incubation period, media was removed and alamarBlue solution $(10 \mu \mathrm{M})$ was added into each treatment well in 1:10 dilution with fresh media. Plates were subsequently incubated at $37{ }^{\circ} \mathrm{C}$ for $4 \mathrm{~h}$, and fluorescence was measured at 540/590 nm using a microplate reader (Synergy HT MultiMode, Biotek, Winooski, VT) for confirmation of cell proliferation after each time point of the study.

\subsection{Data and statistical analyses}

All data are reported as mean \pm standard deviation of at least three replicates. Statistical analyses were done using JMP Pro (Version 10.0.0; SAS Institute Inc., Cary, NC, USA), the differences were considered statistically significant at $p<0.05$. Results were analyzed with two-way ANOVA models with interaction between the independent variables "sample" and "bacterial attachment transmittance" to assess significant differences, followed by Tukey's multiple comparison test $(n=5)$. Asterisks were used to denote statistically highly significant $(* *, p<0.01)$ and significant $(*, p<0.05)$ differences.

\section{Results and discussion}

\subsection{Characterization of RPE-PCL ESNF}

Viscosity of the electrospinning solution is an important parameter since it determines the flow rate and, eventually, the shape of the drop prior to the formation of Taylor's cone. With highly viscous solutions, nanofibers do not form due to the lack of electrical charge strength necessary to promote proper stretching [29]. Since the viscosity of RPE is considerably lower $(\approx 2 \mathrm{mPa} . \mathrm{s})$ than that of PCL $(\approx 136 \mathrm{mPa} . \mathrm{s})$ (Table 1$)$, the addition of RPE has had a negligible effect on the RPE-PCL blends used for electrospinning. However, the addition of RPE enhanced the electrical conductivity of PCL from $1.05 \mu \mathrm{S} / \mathrm{cm}$ to about $1.75 \mu \mathrm{S} / \mathrm{cm}$ (Table 1). This has a beneficial effect as the efficacy of electrospinning and overall ESNF quality (reduction of bead formation) are known to
Table 1

Viscosity and conductivity values for rambutan polyphenol extracts (RPE, concentration $96 \mathrm{mg} / \mathrm{mL}$ ) and the RPE-PCL blends (12 $\mathrm{mg} / \mathrm{mL}: 100 \mathrm{mg} / \mathrm{mL}$ ) used for fabrication of composite electrospun nanofibers. Results are reported as mean $\pm \operatorname{SD}(n=5)$.

\begin{tabular}{lll}
\hline RPE source & Viscosity (mPa.s) & Conductivity $(\mu \mathrm{S} / \mathrm{cm})$ \\
\hline R-134 & $1.87 \pm 0.4$ & $0.52 \pm 0.02$ \\
Rongrein & $2.13 \pm 0.5$ & $0.54 \pm 0.03$ \\
Criollo & $1.87 \pm 0.8$ & $0.51 \pm 0.028$ \\
\hline RPE-PCL Blend & Viscosity (mPa.s) & Conductivity $(\mu \mathrm{S} / \mathrm{cm})$ \\
\hline PCL (no RPE) & $136.7 \pm 5.8$ & $1.05 \pm 0.1$ \\
R-134: PCL & $135.5 \pm 8.9$ & $1.74 \pm 0.4$ \\
Rongrein: PCL & $136.8 \pm 5.6$ & $1.76 \pm 0.8$ \\
Criollo: PCL & $134.3 \pm 5.5$ & $1.72 \pm 0.3$ \\
\hline
\end{tabular}

improve with the conductivity of the feed solution due to jet stretching effect generated by high charges in the solution, which also leads to lowering the diameter ESNF [30]. The viscosity and conductivity values we measured for PCL and RPE-PCL are similar to those reported previously $[31,32]$.

The FTIR-ATR profiles of RPE-PCL ESNF membranes are shown in Fig. 1A. The spectrum of PCL ESNF was different from those of RPE-PCL ESNF, showing the appearance of peaks in different regions. The results do not show any variety-specific differences, with all spectra showing broad phenolic -OH band centered around $3400 \mathrm{~cm}^{-1}$, characteristic -CO stretching at $1665 \mathrm{~cm}^{-1}$, aromatic bending and stretching around 1100 and $1600 \mathrm{~cm}^{-1}$, and $-\mathrm{OH}$ phenolic bending around 1200 and $1400 \mathrm{~cm}^{-1}$. On the other hand, FTIR spectrum of PCL shows a prominent peak at $1730 \mathrm{~cm}^{-1}$, which corresponds to the $-\mathrm{CO}$ (stretching), whereas the peaks at 2868 and $2947 \mathrm{~cm}^{-1}$ are related to the $\mathrm{C}-\mathrm{H}$ bond of saturated carbons. The spectrum of RPE-PCL ESNF showed additional peaks due to the presence of RPE in the blend. The characteristic $1665 \mathrm{~cm}^{-1}$ of RPE's -CO stretching is seen as small shoulder due to the overlapping of the dominant -CO stretching of PCL $\left(1730 \mathrm{~cm}^{-1}\right)$, but the phenolic $-\mathrm{OH}$ corresponding to RPE is seen around $3400 \mathrm{~cm}^{-1}$ for the RPE-PCL ESNF. The spectral analysis did not reveal any rambutan varietal effect on the stability of RPE-PCL ESNF. Similar results were reported by Natarajan et al. [33] for quercetin, a common polyphenol, encapsulated in PCL microspheres.

The incorporation of RPE into PCL ESNF was confirmed by TGA data (Fig. 1B). Thermogram for PCL ESNF show a thermal transition associated with PCL thermal degradation starting around $350{ }^{\circ} \mathrm{C}$. On the other hand, RPE-PCL ESNF showed two thermal transitions, one around 200 ${ }^{\circ} \mathrm{C}$ corresponding to RPE degradation and another around $350{ }^{\circ} \mathrm{C}$, corresponding to PCL degradation. Again, no rambutan varietal differences were observed, with all ESNF showing weight reduction of about $4 \%$, suggesting the efficacy and homogeneity in RPE loading during electrospinning.

Swelling properties of the RPE-PCL ESNF are shown in Fig. 1C. Results indicate the addition of RPE into PCL ESNF significantly improves swelling of the polymeric membranes by about 600 -folds, suggesting RPE increases hydrophilicity and physical performance allowing the membranes to be more flexible and hold higher amounts of water in the swollen 3D nanofiber network.

Release of RPE from ESNF membranes was dependent on the rambutan variety from which RPE was obtained (Fig. 1D). In terms of speed and extent of release, the varieties can be ranked from fast to slow as Rongrein, R-134, and Criollo. However, even Rongerin RPE was released only about $10 \%$ of what was loaded, indicating that most of the RPE remained attached to PCL. The results suggest polyphenolic compounds are released from RPE-PCL ESNFs, considering the specificity of the Folin-Ciocalteu assay for TPC. Previous work [34] has shown that RPE extracted from R-134 variety had higher TPC, 547 $\pm 47 \mathrm{mg}$ gallic acid equivalent (GAE)/g dry mass (DM), whereas TPC values for Criollo and Rongrein varieties were $325 \pm 30$ and $261 \pm 27$ mg GAE/g DM, 
A
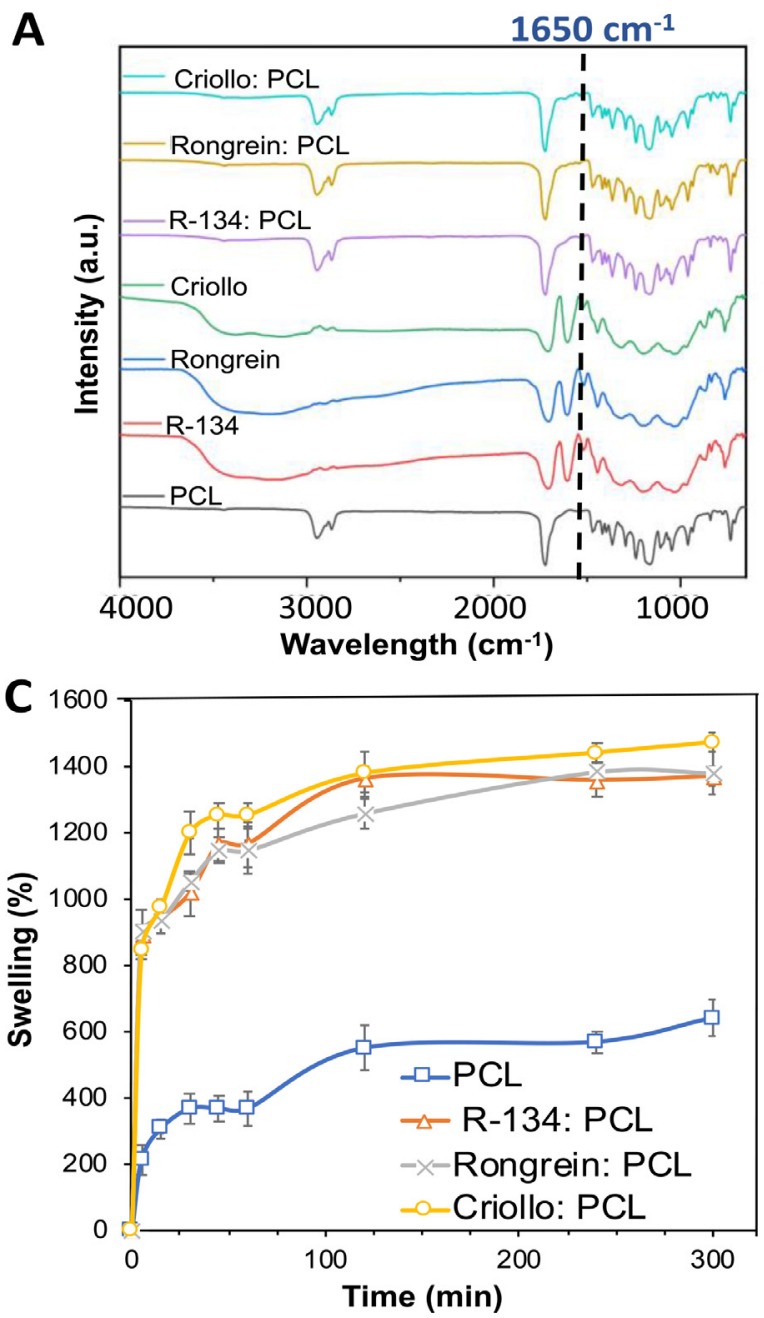

B

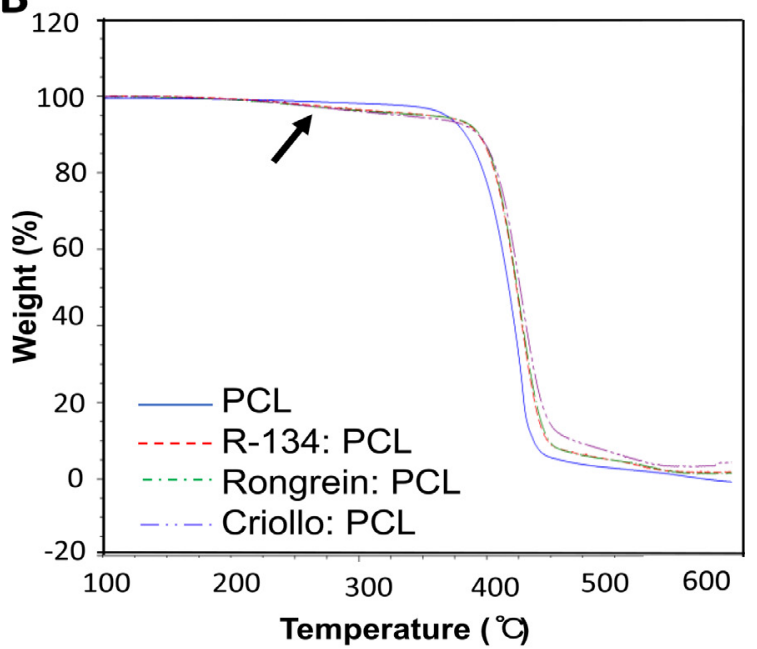

D

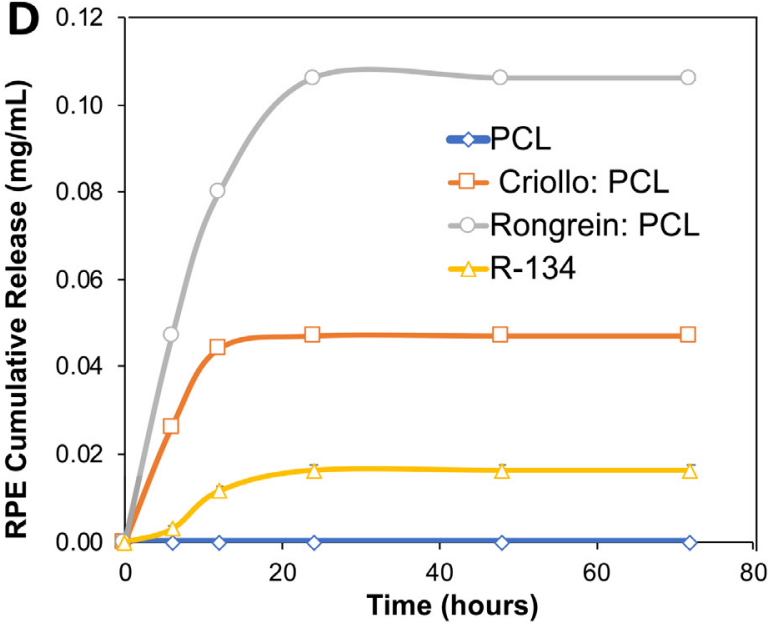

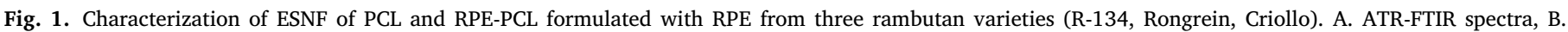

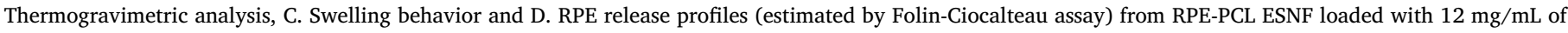
RPE from different varieties of rambutan. All results are expressed as mean $\pm \operatorname{SD}(n=3)$.

Table 2

Average nanofiber diameter and ESNF membrane pore area RPE-PCL ESNF without and with AgNPs decoration. Results are reported as mean $\pm \mathrm{SD}(n=20)$.

\begin{tabular}{lll}
\hline RPE-PCL ESNF & Fiber diameter $(\mathrm{nm})$ & Pore area $\left(\right.$ mean \pm st. dev.) $\left(\mathrm{nm}^{2}\right)$ \\
\hline PCL & $305.29 \pm 68.75$ & $996.94 \pm 106.51$ \\
R-134: PCL & $337.34 \pm 66.86$ & $652.29 \pm 98.69$ \\
Rongrein: PCL & $356.63 \pm 64.29$ & $629.31 \pm 102.47$ \\
Criollo: PCL & $353.81 \pm 97.86$ & $647.17 \pm 85.07$ \\
\hline AgNPs/RPE-PCL ESNF & Fiber diameter $(\mathbf{n m})$ & Pore area (mean \pm st. dev.) $\left(\mathbf{n m}^{2}\right)$ \\
\hline PCL & $419.68 \pm 151.45$ & $898.71 \pm 130.96$ \\
R-134: PCL & $526.12 \pm 99.39$ & $362.03 \pm 84.50$ \\
Rongrein: PCL & $569.49 \pm 125.22$ & $351.15 \pm 123.72$ \\
Criollo: PCL & $594.04 \pm 116.26$ & \pm 119.63 \\
\hline
\end{tabular}

respectively. These results are somewhat similar to $582 \mathrm{mg} \mathrm{GAE} / \mathrm{g} \mathrm{DM}$ value reported for RPE from peels of a Mexican rambutan [35]. Any observed differences are associated with varietal differences, with R-134 showing a slower release, probably due to stronger dipole-dipole interactions in the surface of the fiber owing to its higher TPC and Rongrein showing the fastest release, probably due to its lower TPC and concomitant weaker molecular interactions.
SEM micrographs of the RPE-PCL ESNF (Fig. 2) show fairly uniform fibers with an average diameter of about $300 \mathrm{~nm}$, regardless of the rambutan variety. Results also suggest that, fiber diameter increases with increasing concentration of RPE in the RPE-PCL mixture due to more RPE being incorporated into PCL network, without any varietyspecific effect (Table 2). When compared to PCL ESNF (305 nm), addition of RPE resulted in an average increase of $\sim 50 \mathrm{~nm}$ among the RPE-PCL ESNF. However, the RPE addition drastically decreases the pore area of the ESNF membranes. Also, the diameter of AgNPs/RPEPCL ESNF is much larger due to the hydration of nanofibers when they were dipped in aqueous solutions of $\mathrm{AgNO}_{3}$ and $\mathrm{NaBH}_{4}$, for synthesizing AgNPs. This observation is consistent with the swelling properties of functionalized nanofibers, where RPE increases the hydrophilicity of the polymeric membrane.

\subsection{Characterization of AgNPs/RPE-PCL ESNF}

SEM micrographs of AgNPs/RPE-ESNF show the presence of AgNPs randomly distributed on the nanofiber surface (Fig. 2 insets at the top). Further, AgNPs are more densely distributed on RPE-PCL ESNF than on PCL ESNF, maybe due to higher ion-dipole interactions (i.e., hydrogen bonding) and hydrophilicity of nanofibers containing RPE. Results also suggest the pore size is reduced to about half of the initial mean pore for 

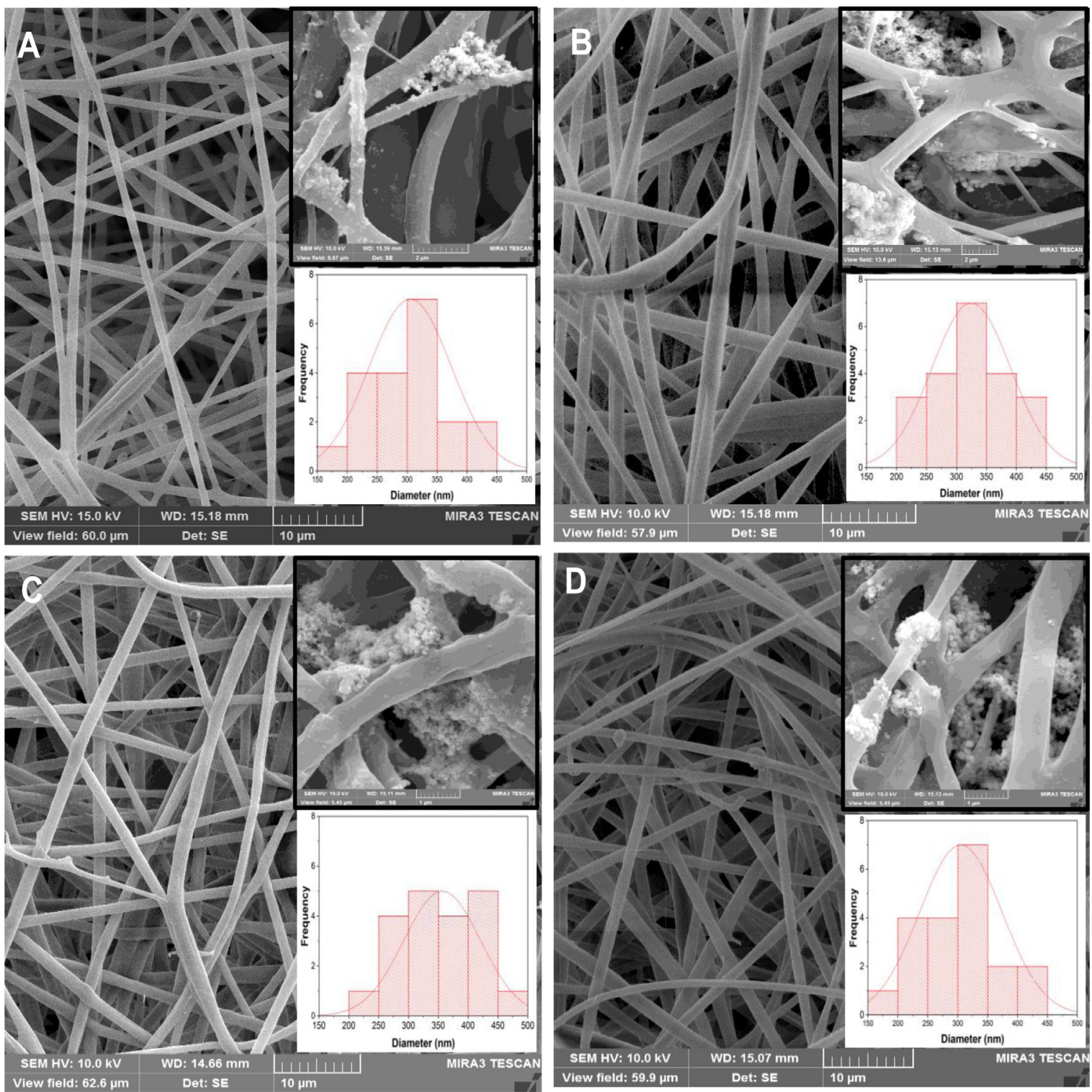

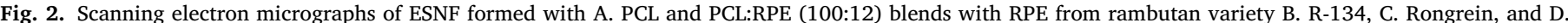

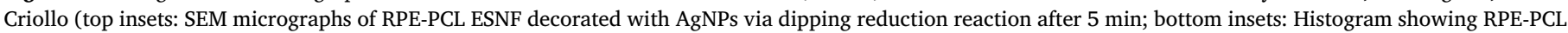
ESNF width distribution as obtained from ImageJ software analysis).

the RPE-loaded ESNF. This reduction can be related to the deposition of the AgNPs, principally in the pores and edges of the ESNF membranes.

Metal nanoparticles, such as Au, Ag, Pt and Pd, which are elemental for catalytic and biomedical applications, can be prepared by adding water soluble reducing agents to aqueous solutions of their respective metal salts in the presence of a stabilizer. The choice of the reducing agent can drastically affect the rates of nucleation and growth of particles, which influence the particle size, size distribution and morphology.

During the AgNPs synthesis, the solution color changed from a colorless at the start of the reaction, to reddish-brown upon the addition of RPE solution, to intense dark-brown when $\mathrm{NaBH}_{4}$ was added at the end of the reaction. These changes in color and hue typically accompny the reduction of silver ions, in our case mediated by the polyphenols in $\mathrm{RPE}$, specially carrying - $\mathrm{CHO}$ and - $\mathrm{OH}$ moieties (Fig. 3A). These compounds are not only electron donors to silver ions, but also coat the surface of nanoparticles for stabilization purposes. These observations are similar to earlier reports [36-38], and are responsible for retaining the decorated AgNPs on the RPE-PCL ESNFs. Fig. 3B is a magnified SEM micrograph of the AgNPs/RPE-PCL ESNF from variety R-134, showing substantial formation AgNPs on nanofibers.

As can be seen in Fig. 3C and 3D, the redox reaction for decorating RPE-PCL ESNFs with AgNPs occurs spontaneously and rapidly catalyzed by the addition of $\mathrm{NaBH}_{4}$. After $1 \mathrm{~min}$ had elapsed, the reaction apparently does not show the presence of AgNPs in suspension, but after 5 min of reaction, the solution darkened considerable, associated with a plasmon increase around $430 \mathrm{~nm}$, indicating the formation of AgNPs, which is easily observed accumulating at the bottom. After $15 \mathrm{~min}$, the reaction has peaked and does not exhibit changes in absorbance. Comparing the previous results with those obtained in this study, it is possible to confirm that the use of the RPE and $\mathrm{NaBH}_{4}$ as reducing and stabilizing agents for decoration of AgNPs allows to carry out the reduction of ions $\mathrm{Ag}^{+}$silver to $\mathrm{Ag}^{0}$ silver nanoparticles rapidly, contrary to long reaction times of 3 to $24 \mathrm{~h}$ reported for RPE alone [36-38]. The absorbance spectra obtained are somewhat asymmetric and some have a very low intensity in the 370-420 nm wavelength range, in which they absorb superficial plasmons of AgNPs. In a reaction time of $3 \mathrm{~h}$, it is possible 
A

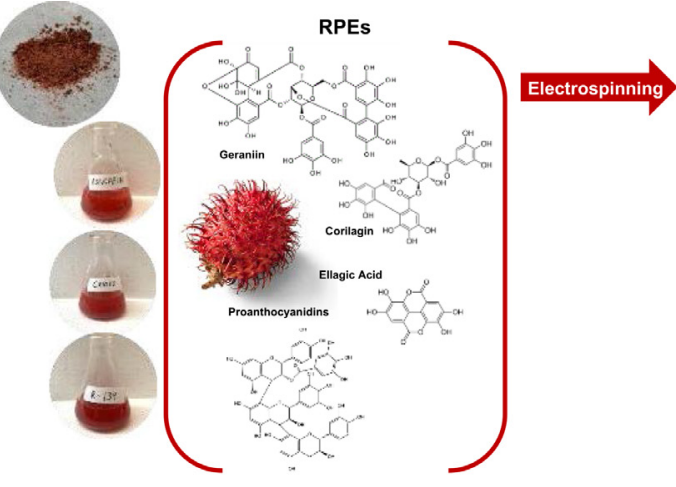

C

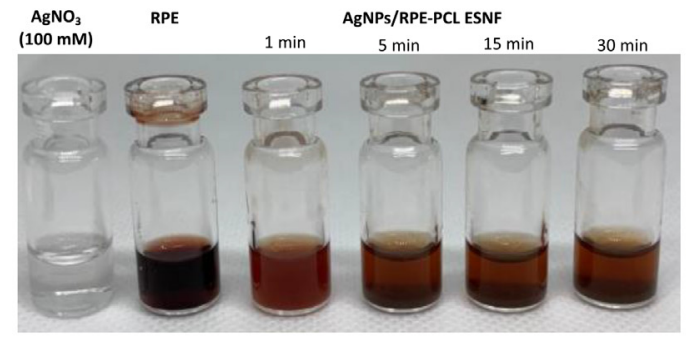

RPE-ESNFs

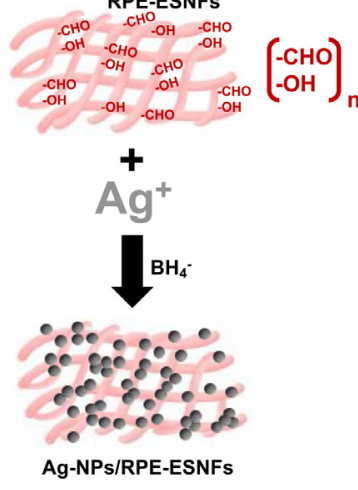

B

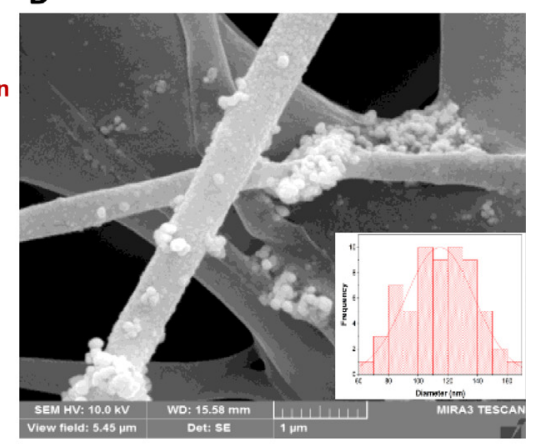

D

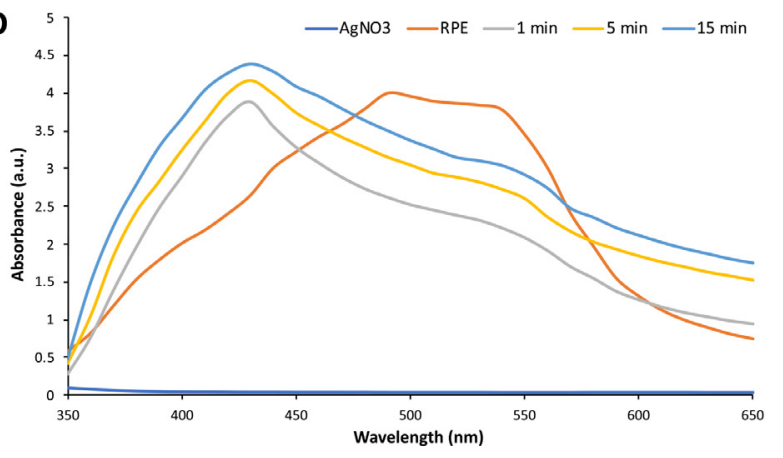

Fig. 3. A. Proposed mechanism for surface decoration of RPE-PCL ESNFs with AgNPs. B. SEM micrograph of AgNPs/RPE-PCL ESNF after 5 min dipping reduction reaction (RPE from variety R-134, scale bar $=1 \mu \mathrm{m}$ ). Inset: histogram distribution showing average particle sizes of AgNPs decorated onto RPE-PCL ESNFs $(n=5)$. C. Picture showing the colors of $\mathrm{AgNO}_{3}, \mathrm{RPE}$, and $\mathrm{AgNO}_{3}$ and $\mathrm{RPE}$ reaction products catalyzed by $\mathrm{BH}_{4}^{-}$at different reaction times (1 to 30 min) and D. corresponding UV-Vis absorption spectra.

to observe the formation of a broadband with a maximum absorption of 0.07 units at $420 \mathrm{~nm}$. After $3 \mathrm{~h}$, the absorbance intensity decreases progressively as the reaction progresses until it stops presenting a maximum. Helmlinger et al. [39] reported this behavior indicating that the formation of unstable particles causes a decrease in the intensity of the absorbance because they disintegrate to form aggregates or large particles with greater stability.

\subsection{Antimicrobial activity of composite ESNFs}

Antibacterial properties of the nanofibers against different pathogenic bacterial strains was evaluated by microbial susceptibility assay, as shown in Fig. 4A-F. ImageJ software was applied for calculating annular radius of each ZoI. At least three sections of each ZoI were calculated for annular radius and the values obtained were used for calculating the percentage of bacterial inhibition in comparison with the positive control (kanamycin $6 \mathrm{mg} / \mathrm{mL}$ ), as shown in Fig. 5 . Results are in agreement with qualitative observation of ZoIs, showing a statistically significant increase $(p<0.05)$ in antibacterial activity for all RPE-loaded ESNF when compared to control PCL ESNF. Among different rambutan varieties tested, only Rongrein sample showed slightly lower bioactivity $(p<0.05)$ against all bacterial strains tested. These results are in agreement with the release profiles obtained for different varieties of rambutan and also related to their TPC as discussed previously. Phuong et al. [40] also reported antibacterial (against Gram-positive and Gram-negative) properties of RPEs supporting the bioactivity of polyphenolic compounds such as geraniin, ellagic acid, rutin, quercetin, and corilagin.

The effect of RPE against bacterial growth was higher in conjunction with AgNPs. Moreover, antibacterial activity of AgNPs/RPE-PCL ESNF was significantly higher than that of RPE/PCL ESNF, suggesting a synergistic effect between AgNPs and RPE from all rambutan varieties, though the effect of RPE of R-134 was higher than the one from Rongrein and Criollo. Overall moderate (30 to 50\%) to high (>60\%) bacterial inhibition compared with positive control (kanamycin, 100\% inhibition) suggest that RPE addition can improve the use of ESNF membranes for potential antibacterial coating or as wound healing template.

Similar behavior was reported by Shrivastava et al. [41], who assumed that the differences between Gram-positive and Gstaphramnegative bacteria reside in the structure of their cell walls, where both RPE and AgNPs interact. Gram-negative bacteria, such as E.coli, have an outer layer of lipopolysaccharides that lacks strength and rigidity, where the negative charges of lipopolysaccharides are attracted to the weak charges available on AgNPs. However, net charge on AgNPs are reduced due to its interaction with RPE among the surface of the ESNFs, depicting a weaker interaction with the Gram-negative bacteria, as also observed for P. aeruginosa. On the other hand, the cell wall of Gram-positive bacteria such as $S$. aureus, is composed of a layer of peptidoglycan with a greater thickness of 20-80 nm, which consists of linear polysaccharide chains that are cross-linked with short peptides, providing greater rigidity and suitable for stronger intermolecular interaction via hydrogen bonding and dipole-dipole interactions that increase the anchorage sites for AgNPs and RPEs with the bacterial cell wall. For this reason, it can be said that the AgNPs/RPE-PCL ESNFs had a lower bactericidal effect against $E$. coli and $P$. aeruginosa than against $S$. aureus.

\subsection{Cell adhesion and proliferation studies on composite ESNFS}

All RPE-PCL ESNF showed a highly significant increase in cell adhesion $(p<0.01)$ when compared to that of PCL ESNF (Fig. 6A), suggesting the addition of RPE significantly improves biocompatibility. RPE seems to provide a suitable microenvironment for cell adhesion, the first step for key biological processes such as wound healing and tissue regenera- 

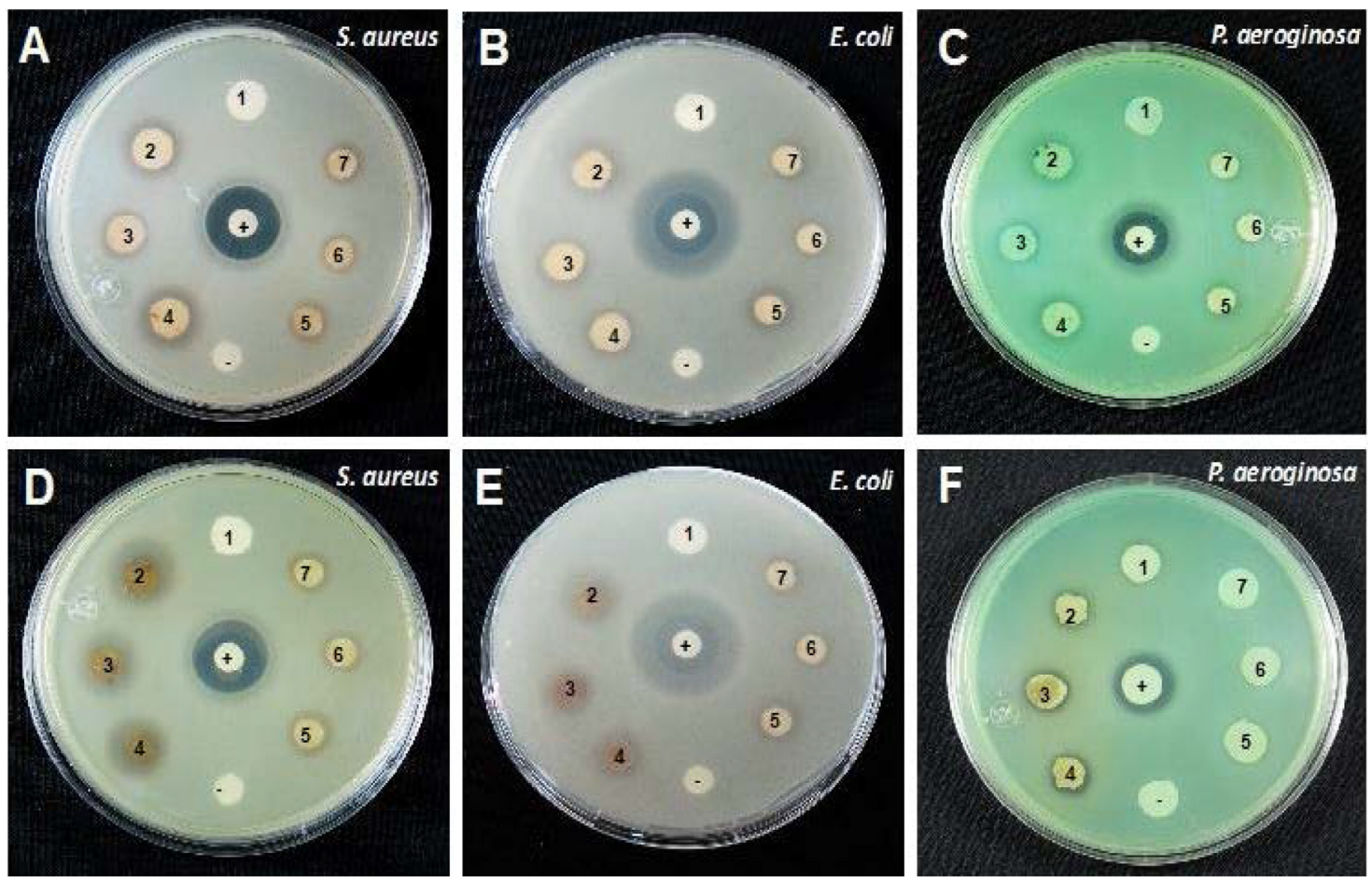

Fig. 4. Antibacterial activity, measured as zone-of-inhibition, against three bacterial pathogens: $E$. coli, $S$. aureus, and $P$. aeruginosa, after $24 \mathrm{~h}$ incubation. A-C. RPE-PCL ESNF, D-F. AgNPs/RPE-PCL ESNF (Code: 1 PCL (no RPE added); 2 R-134: PCL ESNFs; 3 Rongrein: PCL ESNFs; 4 Criollo: PCL ESNFs; 5 R-134 (alone); 6 Rongrein (alone); 7 Criollo (alone); Negative control (no treatement) and positive control (kanamycin $6 \mathrm{mg} / \mathrm{mL}$ )).

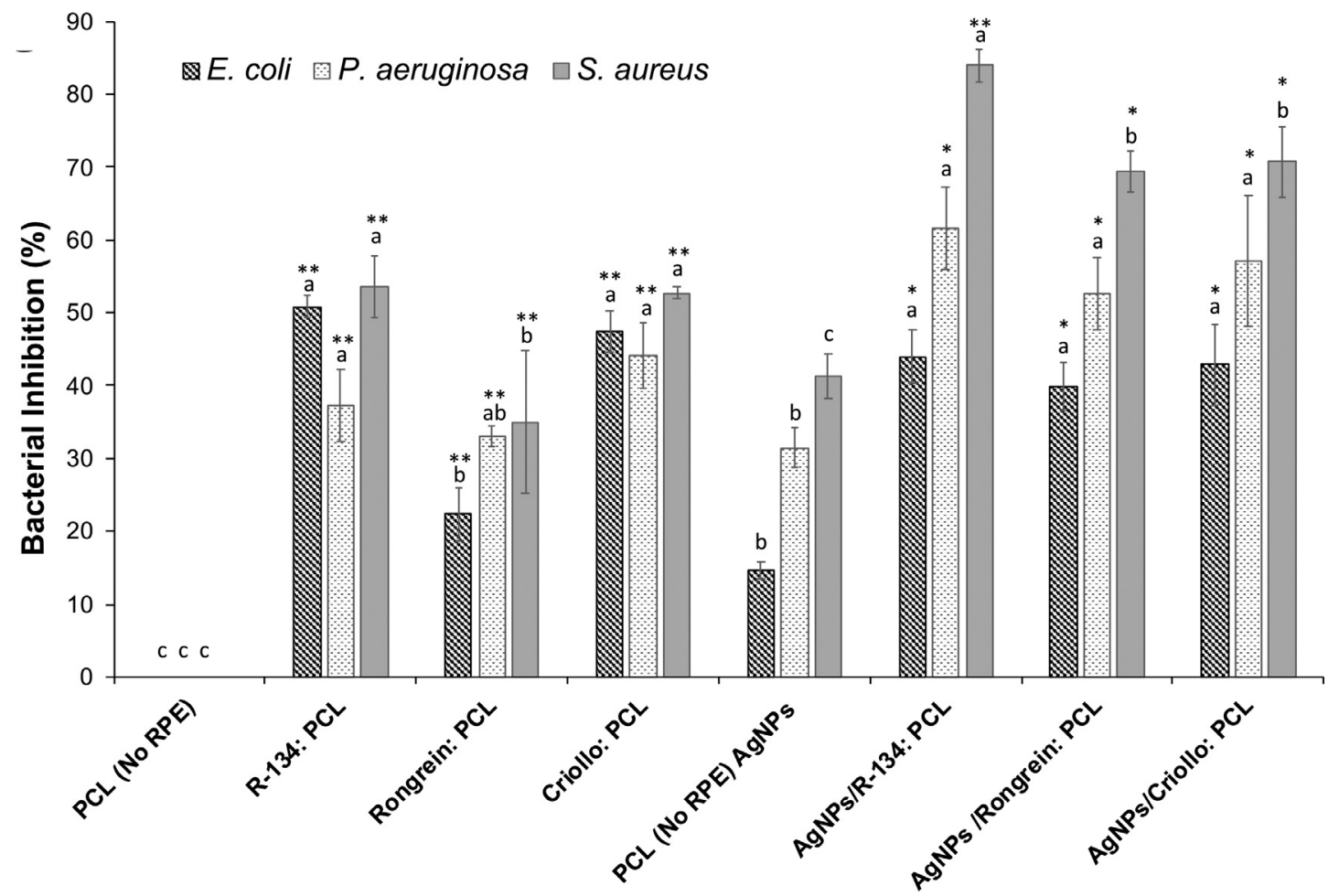

Fig. 5. Antimicrobial activity of RPE-PCL ESNF with and without AgNPs, after $24 \mathrm{~h}$ of bacterial growth. All results are expressed as mean \pm SD $(n=3) .{ }^{*}=\operatorname{statistically}$ significant at $p<0.05$ and ${ }^{* *}=$ statistically significant at $p<0.01$. Bars with different letters are significantly different among each independent system $(p<0.05$, $n=3)$. 

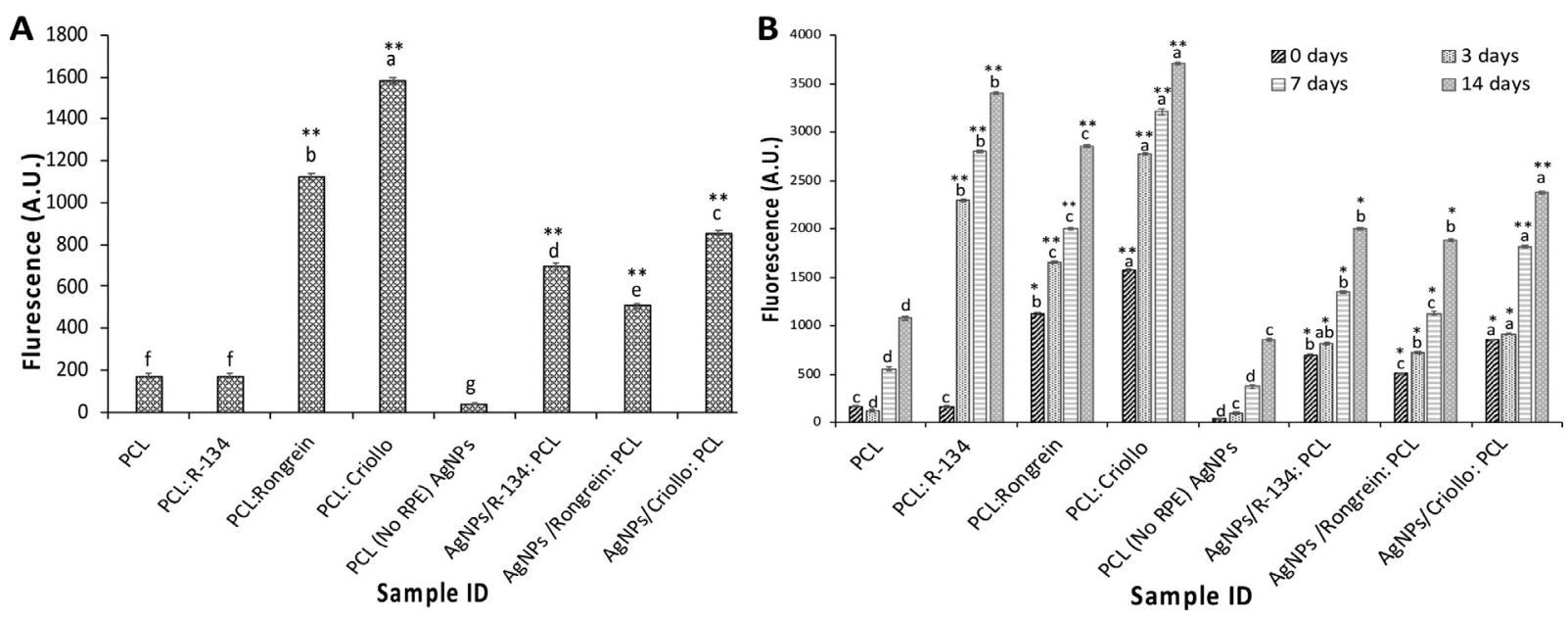

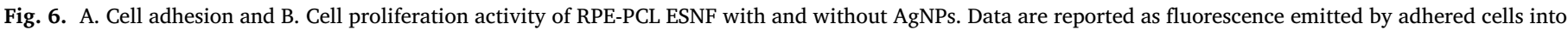

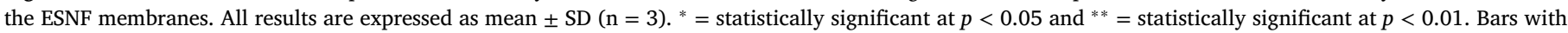
different letters are significantly different among each independent system, RPE-PCL ESNFs and AgNPs/RPE-PCL ESNFs, respectively ( $p<0.05$, $n=3$ ).

tion. Additionally, ESNF loaded with RPEs from Criollo variety showed slightly higher bioactivity than the other two rambutan varieties tested.

On the other hand, AgNPs/RPE-PCL ESNF showed lower cell adhesion than the systems containing only RPEs, suggesting addition of AgNPs hinders cell adhesion, maybe due to a blocking effect of the functional groups in RPEs that promotes adhesion of cells on the surface of the ESNF. However, when compared to the AgNPs/PCL ESNF control, hybrid systems containing RPEs showed a highly significant increase ( $p$ $<0.01$ ) in cell adhesion for varieties R-134 and Criollo and a significant increase $(p<0.05)$ for variety Rongrein, showing a similar trend than the one observed for RPE-ESNF without AgNPs. The lower biocompatibility exhibited by Rongrein RPE may be attributed to its low retention in the ESNF network (Fig. 6A).

These results suggest the functionalization of synthetic ESNF membranes with RPE highly significantly $(p<0.01)$ enhances cell proliferation (Fig. 6B). Similar to the results obtained in cell adhesion experiments, RPEs from variety Rongrein showed slightly lower cell proliferation than varieties Criollo and R-134, maybe due to smaller cell count initially attached to the RPE-ESNF membrane during adhesion stage. Likewise, coating of RPE-ESNF with AgNPs did not positively impact bioactivity of hybrid ESNF membranes for cell proliferation. Incorporation of RPEs into hybrid systems containing AgNPs still showing a highly significant improvement on cell proliferation $(p<0.01)$ for all rambutan varieties in the time intervals of incubation tested, reinforcing our original hypothesis that functionalization of synthetic ESNF membranes with bioactive plant-based extracts from rambutan will significantly improve biocompatibility, cell attachment and proliferation properties, becoming a suitable system for nanocoating of poorly biocompatible biomaterials.

Some studies have shown that the intrinsic toxicity of AgNPs depend on a range of factors such as, size, shape, surface area, surface charge, solubility and state of agglomeration. In addition, a number of in vitro studies indicate that AgNPs are toxic to mammalian cells [42]. As with other nanoparticles, the toxicity of AgNPs seems to be associated with their oxidative and inflammatory nature, which generates genotoxicity and cytotoxicity $[43,44]$. The generation of reactive oxygen species (ROS) and oxidative stress seem to be the two main mechanisms of toxicity [45]. There must be a balance in the body between ROS and antioxidant defense systems. When this balance is broken in favor of the ROS, the so-called oxidative stress or damage occurs [46]. Thus, more experiments are needed to optimize AgNPs deposition reaction, by taking advantage of the green redox reaction between $\mathrm{RPEs}$ and $\mathrm{Ag}^{+}$, avoid- ing the need for chemical catalyzer and possibly leading to smaller, less agglomerated and lower toxicity AgNPs decorated onto RPE-PCL ESNFs.

\section{Conclusions}

We fabricated RPE-loaded nanofibers via electrospinning. The efficacy of RPE loading into the nanofibers was confirmed by spectroscopy (FTIR), thermal analysis (TGA), electron microscopy (SEM), and swelling studies, suggesting functionalization of synthetic ESNF with RPEs will increase average nanofiber size, reduce overall membrane pore area and increase hydrophilicity. Swelling and release studies conducted in RPE-loaded ESNF showed that RPEs could be successfully entrapped in the ESNF network, promoting effective functionalization of synthetic ESNF. RPE-loaded ESNF succeeded in improving antibacterial, cell adhesion and cell proliferation properties of synthetic ESNF membranes. A combination of RPEs with AgNPs showed to be effective for providing a hybrid ESNF system exposing a synergistic behavior against pathogenic bacteria, indicating that RPEs significantly improved well-known properties of AgNPs as a broad-spectrum antibacterial agent.

\section{Declaration of Competing Interest}

Authors declare no conflict of interests.

\section{Acknowledgments}

We acknowledge the support from Costa Rican Ministerio de Ciencia Tecnologia y Telecomunicaciones (MICITT) and Consejo Nacional para Investigaciones Científicas y Tecnologicas (CONICIT). We gratefully thank Prof. Belkis Sulbaran and Dr. Hasbleidy Palacios from Centro Cientifico Tonala, Universidad de Guadalajara, Mexico for their support with scanning electron microscopy.

\section{References}

[1] E. Miser-Salihoglu, G. Akaydin, E. Caliskan-Can, S. Yardim-Akaydin, Evalution of antioxidant activity of various herbal folk medicines, J. Nutrit. Food Sci. 3 (5) (2013).

[2] P. Krishnan, The scientific study of herbal wound healing therapies: Current state of play, Current Anaesthesia Critical Care 17 (1-2) (2006) 21-27.

[3] B.P. Nagori, R. Solanki, Role of medicinal plants in wound healing, Res. J. Med. Plants 5 (4) (2011) 392-405.

[4] G. Thilagavathi, S.K. Bala, Microencapsulation of plant-based extracts for microbial resistance in healthcare textiles, Indian J. Fiber Textile Res. 32 (2007) 351-354. 
[5] S.A. Wang, M.F. Marcone, S. Barbut, L.T. Lim, Electrospun soy protein isolate-based fiber fortified with anthocyanin-rich red raspberry (Rubus strigosus) extracts, Food Res. Int. 52 (1) (2013) 467-472.

[6] S. Madrigal-Carballo, L. Haas, M. Vestling, C.G. Krueger, J.D. Reed, Non-covalent pomegranate (Punica granatum) hydrolyzable tannin-protein complexes modulate antigen uptake, processing and presentation by a T-cell hybridoma line co-cultured with murine peritoneal macrophages, Int J Food Sci. Nutr. 67 (8) (2016) 960-968.

[7] H. Urena-Saborio, E. Alfaro-Viquez, D. Esquivel-Alvarado, S. Madrigal-Carballo, S. Gunasekaran, Electrospun plant mucilage nanofibers as biocompatible scaffolds for cell proliferation, Int. J. Biol. Macromol. 115 (2018) 1218-1224.

[8] K. Moomand, L.T. Lim, Properties of Encapsulated Fish Oil in Electrospun Zein Fibres Under Simulated In Vitro Conditions, Food Bioprocess Tech. 8 (2) (2015) 431-444.

[9] P. Wen, D.H. Zhu, H. Wu, M.H. Zong, Y.R. Jing, S.Y. Han, Encapsulation of cinnamon essential oil in electrospun nanofibrous film for active food packaging, Food Control 59 (2016) 366-376.

[10] N.M. Hani, A.E. Torkamani, M.H. Azarian, K.W.A. Mahmood, S.H. Ngalim, Characterisation of electrospun gelatine nanofibres encapsulated with Moringa oleifera bioactive extract, J. Sci. Food Agr. 97 (10) (2017) 3348-3358.

[11] V. Guarino, V. Cirillo, L Ambrosio, Bicomponent electrospun scaffolds to design extracellular matrix tissue analogs, Expert Rev. Med. Devic 13 (1) (2016) 83-102.

[12] N. Thitileadecha, A. Teerawutgulrag, N. Rakariyatham, Antioxidant and antibacterial activities of Nephelium lappaceum L. extracts, Lwt-Food Sci. Technol. 41 (10) (2008) 2029-2035.

[13] M. Arias, I. Cavo, El cultivo de rambután (Nephelium lappaceum L.), Ed. MAG/INTA/FITTACORI, San Jose, Costa Rica, 2014.

[14] U.D. Palanisamy, L.T. Ling, T. Manaharan, D. Appleton, Rapid isolation of geraniin from Nephelium lappaceum rind waste and its anti-hyperglycemic activity, Food Chem. 127 (1) (2011) 21-27.

[15] S. Elendran, L.W. Wang, R. Prankerd, U.D. Palanisamy, The physicochemical properties of geraniin, a potential antihyperglycemic agent, Pharm Biol. 53 (12) (2015) 1719-1726.

[16] W.J. Liang, Q.Y. Ma, H.Z. Jiang, J. Zhou, J. Pang, Y.X. Zhao, A new hederagenin glycoside from Nephelium lappaceum, Chem. Nat. Compd. 47 (6) (2012) 935-939.

[17] C.L. Keat, A. Aziz, A.M. Eid, N.A. Elmarzugi, Biosynthesis of nanoparticles and silver nanoparticles volume 2, Article number: 47 (2015) C, Bioresources Bioproces. 2 (2015).

[18] M.A. Radzig, V.A. Nadtochenko, O.A. Koksharova, J. Kiwi, V.A. Lipasova, I.A. Khmel, Antibacterial effects of silver nanoparticles on gram-negative bacteria: Influence on the growth and biofilms formation, mechanisms of action, Colloid Surface B 102 (2013) 300-306.

[19] W.R. Li, X.B. Xie, Q.S. Shi, H.Y. Zeng, Y.S. Ou-Yang, Y.B. Chen, Antibacterial activity and mechanism of silver nanoparticles on Escherichia coli, Appl. Microbiol. Biot. 85 (4) (2010) 1115-1122.

[20] K.H. Hong, J.L. Park, I.H. Sul, J.H. Youk, T.J. Kang, Preparation of antimicrobial poly(vinyl alcohol) nanofibers containing silver nanoparticles, J. Polym Sci. Pol, Phys. 44 (17) (2006) 2468-2474.

[21] Y.G. Wu, W.J. Jia, Q. An, Y.F. Liu, J.C. Chen, G.T. Li, Multiaction antibacterial nanofibrous membranes fabricated by electrospinning: an excellent system for antibacterial applications, Nanotechnology 20 (24) (2009).

[22] S.D. Solomon, M. Bahadory, A.V. Jeyarajasingam, S.A. Rutkowsky, C. Boritz, L. Mulfinger, Synthesis and study of silver nanoparticles, J. Chem. Educ. 84 (2) (2007) 322-325.

[23] N.A. Hotaling, K. Bharti, H. Kriel, C.G. Simon, DiameterJ: A validated open source nanofiber diameter measurement tool, Biomaterials 61 (2015) 327-338.

[24] V.L. Singleton, R. Orthofer, R.M. Lamuela-Raventos, Analysis of total phenols and other oxidation substrates and antioxidants by means of Folin-Ciocalteu reagent, Method Enzymol. 299 (1999) 152-178.

[25] V. Bondet, W. BrandWilliams, C. Berset, Kinetics and mechanisms of antioxidant activity using the DPPH* free radical method, Food Sci. Technol. Leb 30 (6) (1997) 609-615.

[26] C. Spagnol, E.H. Fragal, A.G.B. Pereira, C.V. Nakamura, E.C. Muniz, H.D.M. Follmann, R. Silva, A.F. Rubira, Cellulose nanowhiskers decorated with silver nanoparticles as an additive to antibacterial polymers membranes fabricated by electrospinning, J. Colloid Interf. Sci. 531 (2018) 705-715.
[27] J. Vivas, D. Garzón-Alvarado, M. Cerrolaza, Modeling cell adhesion and proliferation: a cellular-automata based approach, Adv. Model. Simul. Eng. Sci. 2 (1) (2015) 32.

[28] P. Rejmontova, Z. Capakova, N. Mikusova, N. Marakova, V. Kasparkova, M. Lehocky, P. Humpolicek, Adhesion, Proliferation and Migration of NIH/3T3 Cells on Modified Polyaniline Surfaces, Int. J. Mol. Sci. 17 (9) (2016).

[29] S.L. Shenoy, W.D. Bates, H.L. Frisch, G.E. Wnek, Role of chain entanglements on fiber formation during electrospinning of polymer solutions: good solvent, non-specific polymer-polymer interaction limit, Polymer 46 (10) (2005) 3372-3384.

[30] X.H. Zong, K. Kim, D.F. Fang, S.F. Ran, B.S. Hsiao, B. Chu, Structure and process relationship of electrospun bioabsorbable nanofiber membranes, Polymer 43 (16) (2002) 4403-4412.

[31] Y.F. Li, M. Rubert, H. Aslan, Y. Yu, K.A. Howard, M.D. Dong, F. Besenbacher, M.L. Chen, Ultraporous interweaving electrospun microfibers from PCL-PEO binary blends and their inflammatory responses, Nanoscale 6 (6) (2014) 3392-3402.

[32] R. Ramalingam, C. Dhand, C.M. Leung, S.T. Ong, S.K. Annamalai, M. Kamruddin, N.K. Verma, S. Ramakrishna, R. Lakshminarayanan, K.D. Arunachalam, Antimicrobial properties and biocompatibility of electrospun poly-epsilon-caprolactone fibrous mats containing Gymnema sylvestre leaf extract, Mat. Sci. Eng. C-Mater. 98 (2019) 503-514.

[33] V. Natarajan, N. Krithica, B. Madhan, P.K. Sehgal, Formulation and evaluation of quercetin polycaprolactone microspheres for the treatment of rheumatoid arthritis, J. Pharm Sci. Us 100 (1) (2011) 195-205.

[34] H. Rojas, Evaluaciòn de la presencia de actividad antioxidante y antimicrobiana de los taninos condensados en el exocarpio de siete variedades de rambután, (Nephelium lappaceum L.), School of Chemistry, National University, Heredia, Costa Rica, 2016.

[35] C. Hernandez, J. Ascacio-Valdes, H. De la Garza, J. Wong-Paz, C.N. Aguilar, G.C. Martinez-Avila, C. Castro-Lopez, A. Aguilera-Carbo, Polyphenolic content, in vitro antioxidant activity and chemical composition of extract from Nephelium lappaceum L. (Mexican rambutan) husk, Asian Pac. J. Trop. Med. 10 (12) (2017) 1201-1205.

[36] B. Kumar, K. Smita, Y. Angulo, L. Cumbal, Valorization of rambutan peel for the synthesis of silver-doped titanium dioxide ( $\mathrm{Ag} / \mathrm{TiO} 2)$ nanoparticles, Green Process Synth. 5 (4) (2016) 371-377.

[37] P. S., T. Norasingsatorn, N. Yongvanich, Effect of $\mathrm{pH}$ on the environmentally friendly fabrication of silver nanoparticles using rambutan peel extract, Key Eng. Mater 824 (2019) 149-155.

[38] B. Kumar, K. Smita, L. Cumbal, Y. Angulo, Fabrication of silver nanoplates using Nephelium lappaceum (Rambutan) peel: A sustainable approach, J. Mol. Liq. 211 (2015) 476-480.

[39] J. Helmlinger, C. Sengstock, C. Gross-Heitfeld, C. Mayer, T.A. Schildhauer, M. Koller, M. Epple, Silver nanoparticles with different size and shape: equal cytotoxicity, but different antibacterial effects, Rsc. Adv. 6 (22) (2016) 18490-18501.

[40] N.N. Phuong, T.T. Le, J.V. Camp, K. Raes, Evaluation of antimicrobial activity of rambutan (Nephelium lappaceum L.) peel extracts, Int. J. Microbiol. 321 (108539) (2020).

[41] S. Shrivastava, T. Bera, A. Roy, G. Singh, P. Ramachandrarao, D. Dash, Characterization of enhanced antibacterial effects of novel silver nanoparticles, Nanotechnology 18 (22) (2007)

[42] O. Choi, Z.Q. Hu, Size dependent and reactive oxygen species related nanosilver toxicity to nitrifying bacteria, Environ Sci. Technol. 42 (12) (2008) 4583-4588.

[43] P.V. Asharani, Y.L. Wu, Z.Y. Gong, S. Valiyaveettil, Toxicity of silver nanoparticles in zebrafish models, Nanotechnology 19 (25) (2008).

[44] S.M. Hussain, K.L. Hess, J.M. Gearhart, K.T. Geiss, J.J. Schlager, In vitro toxicity of nanoparticles in BRL 3A rat liver cells, Toxicol. Vitro 19 (7) (2005) 975-983.

[45] Y.H. Hsin, C.F. Chen, S. Huang, T.S. Shih, P.S. Lai, P.J. Chueh, The apoptotic effect of nanosilver is mediated by a ROS- and JNK-dependent mechanism involving the mitochondrial pathway in NIH3T3 cells, Toxicol Lett. 185 (2) (2009) 142-142(vol 179, pg 130, 2008).

[46] A. Nyska, R. Kohen, Oxidation of biological systems: Oxidative stress phenomena, antioxidants, redox reactions, and methods for their quantification, Toxicol Pathol. 30 (6) (2002) 620-650. 